\title{
A Variational Model for Histogram Transfer of Color Images
}

\author{
N. Papadakis ${ }^{1}$, E. Provenzi ${ }^{2}$, and Vicent Caselles $^{2}$ \\ ${ }^{1}$ CNRS, LJK, UMR 5224, Grenoble, France \\ ${ }^{2}$ Departament de Tecnologies de la Informació i les Comunicacions ,UPF - \\ Universitat Pompeu Fabra, Barcelona
}

\begin{abstract}
In this paper we propose a variational formulation for intermediate color histogram equalization of two or more images. We study an energy functional composed by three terms: one tends to approach the cumulative histograms of the original images, the other two tends to maintain the initial colors and geometry. By minimizing this energy, we obtain an algorithm that balances equalization and conservative features. As a result, the original images evolve approaching the intermediate histogram between the original images. As a novel result, this midway histogram does not need to be specified in advance, but it is a natural result of the model. Finally, we provide experiments showing that the proposed method compares well with the state of the art.
\end{abstract}

\section{INTRODUCTION}

This paper is devoted to a variational formulation of color grading, that is the color matching of two or more images. We will show that, by minimizing a suitable energy functional, images will be matched to an intermediate common histogram.

Color grading is a main tool for color matching in the post-production industry and its need arises in many applications. For example, the same scene can be photographed with different cameras or under different illumination conditions, resulting in images that may have quite different colors. Other noticeable examples are given by flicker stabilization of video sequences and color balancing, a process that is mostly done manually. 
On the other hand, color grading plays an important role also in one of the main topics in computer vision: the numerical comparison of images in order to correlate them or extract mutual information. Applications can be found in different contexts like depth or disparity computation in stereo images, or optical flow computation. Due the use of different cameras or to the variability of light conditions, a color normalization may be an important step previous to the numerical image comparison.

The main approach to color grading is based on the computation of a color transfer map. Given two images, a source and a target, the problem is to find a mapping that transforms the source into an image with a color statistics that is as similar as possible to that of the target. For some applications, instead of a target image, one might want to specify a reference palette to be matched. The modified image should keep the geometry of the original one, only its color aspect should be modified.

In this paper we propose a new variational model to transform two or more color images in order to approach their color histograms as much as possible. To briefly describe the process, let us consider here the case of two given color images $\vec{U}, \vec{V}$ : we want to transform them into new images $\vec{u}$ and $\vec{v}$ that approximately share an intermediate color histogram which is not specified in advance and, at the same time, we want to maintain the geometry of $\vec{U}$ and $\vec{V}$, respectively. Thus, the energy has to combine the comparison of histograms with color and shape constraints (which can be codified by the gradient direction). The shape constraints can be related to the filtering process constrained by the original gradient field used in [6].

Let us remark that the mapping to an intermediate histogram permits to distribute the errors in the histogram matching process without privileging one particular image with respect to the others. Up to our knowledge, there is no method in the literature that allows realizing such a process without defining the histogram beforehand.

The plan of the paper is the following. In Section II we provide a review of the state of the art in color grading and midway histogram equalization, emphasizing the role played by the Monge-Kantorovich mass transportation theory. As a technical tool, in Section III we define the $L^{2}$ distance between the cumulative histogram of an image $I$ and a reference one and we compute its first variation with respect to $I$. In Section IV we describe our variational model for intermediate color histogram equalization of a given set of color images. The model penalizes the histogram separation of the set of images and constrains each of them to preserve its color and 
geometric information. In Section V we describe the algorithm we use to minimize the proposed energy. In Section VI we present some experiments that illustrate the model and compare it with the results obtained using the algorithm proposed in [13]. Finally, in Section VII we summarize our main conclusions.

\section{REVIEW OF THE STATE OF THE ART IN COLOR GRADING AND MIDWAY HISTOGRAM EQUALIZATION}

As previously commented, the central problem in color grading is to find a suitable transfer map. This function can be computed to match simple statistical values, like mean and variance [1], the covariance matrix [2], or the whole color histogram [3]-[6]. The matching of statistics can thus be done separately for each channel in the $l \alpha \beta$ color space which has uncorrelated components as in [1]. Another possibility is to compute an affine map [7]-[9] to map the covariance matrices of the two color images. Concerning the map of color histograms, several works have also been proposed, for instance, in [10], matching of color histograms is done on the three channels independently. The color histogram equalization can also be performed by computing a transfer map using mesh deformations to fit the existing histogram to a uniform histograms [11].

The formulation of color histogram matching in terms of Monge-Kantorovich's mass transportation theory has been considered in [3], [12], [13]. In [3], [12] the authors compute a transport plan (also called transference plan, see [14]) matching two histograms which minimizes the total displacement cost measured in terms of the Earth Mover Distance (EMD) (also called Wasserstein distance [14]) and solve this problem using linear programming.

This technique has been applied in [12] to midway color histogram equalization (see [15], [16] for the case of gray level images), that is, to the problem of finding a midway histogram in the sense of EMD distance [14] and computing mappings that transform the original images into images that share this histogram.

In [13], assuming that the histograms of two given color images can be approximated by multivariate Gaussian distributions, Monge-Kantorovich's formulation is restricted to find a linear map that approximates the transfer of histograms. In practice, this method is applied to transfer the histogram of one image to another although it could be used to define a midway histogram as in [12], [15]. 
The transfer map in the color grading method proposed in [6] is computed by iteratively equalizing all principal axis marginals computed for every possible rotation of the histogram, until convergence. Then the method is refined by a filtering process, constrained by the original gradient field, to reduce possible grain artifacts [6].

For later convenience, let us remind here a remark about midway histogram equalization pointed out by Delon [15]: if $H_{0}, H_{1}$ are the cumulative histograms of two original gray-scale images $I_{0}$ and $I_{1}$, respectively, then mapping them to have a cumulative histogram which is the direct average of the original ones, i.e. $\tilde{H}:=\left(H_{0}+H_{1}\right) / 2$, is not always a suitable choice for midway equalization. An illuminating counterexample is given by the case when $I_{0}$ and $I_{1}$ are unimodal images with spread modes centered at the levels $\lambda_{0}$ and $\lambda_{1}$, respectively, with $\lambda_{0} \neq \lambda_{1}$. Ideally, one would expect a midway image to present a single mode centered at $\left(\lambda_{0}+\lambda_{1}\right) / 2$, instead it is clear that the composition of $I_{0}$ with $\tilde{H}^{-1} \circ H_{0}$ spreads the gray levels of the image on two modes centered at $\lambda_{0}$ and $\lambda_{1}$ in order to make its histogram correspond as much as possible to $\tilde{H}$. Thus, it generates new structures that were not present before [12], [15]. Instead, the natural intermediate histogram $\bar{H}$ is given by

$$
\bar{H}:=\left(\frac{H_{0}^{-1}+H_{1}^{-1}}{2}\right)^{-1} .
$$

Let $\psi_{0}:=\bar{H}^{-1} \circ H_{0}$ and $\psi_{1}:=\bar{H}^{-1} \circ H_{1}$ be the mappings of images $I_{0}$ and $I_{1}$ into images that share the midway histogram $\bar{H}$. Defining $T:=H_{1}^{-1} \circ H_{0}$, we have

$$
\psi_{0}=\frac{I+H_{1}^{-1} \circ H_{0}}{2}=\frac{I+T}{2}, \quad \psi_{1}=\frac{I+H_{0}^{-1} \circ H_{1}}{2}=\frac{I+T^{-1}}{2}
$$

and $\bar{H}=\left(\frac{I+T}{2}\right)^{-1} \circ H_{0}=H_{1} \circ\left(\frac{I+T^{-1}}{2}\right)^{-1}$. Observe that $T$ maps $I_{0}$ into an image whose histogram is $H_{1}$. In [16] $\psi_{0}, \psi_{1}$ are computed using a dynamic programming algorithm.

Delon shows that these results can be embedded in a more abstract Monge-Kantorovich's framework which has the advantage to be easily extendible to any dimension, thus permitting to analyze color images. Let $h_{0}, h_{1}$ be the histograms of two generic images $I_{0}, I_{1}$, then $h_{0}, h_{1}$ can be seen as the Lebesgue densities of the probability measures $d \mu_{0}(x)=h_{0}(x) d x, d \mu_{1}(x)=h_{1}(x) d x$ describing the distribution of the image values. The search of an intermediate histogram between $h_{0}$ and $h_{1}$ is then equivalent to the search of an intermediate measure between $\mu_{0}$ and $\mu_{1}$. It can be proven that $\bar{H}$ can be seen as the cumulative distribution function of the middle point $\mu_{\frac{1}{2}}$ of the geodesic that connects $\mu_{0}$ and $\mu_{1}$ in the space of probability measures with respect to the 
quadratic transportation cost [14], [15]. As already observed, the discrete Monge-Kantorovich problem gives a transport plan that requires the solution of a linear programming problem [3], [12] to be computed. Let us mention that transport plans permit to split the mass of a bin of a given image histogram and distribute it between several bins of the other histogram. This may be considered a disadvantage. Moreover, the transport plan has the dimension of the product between the number of bins in both the original and the target image, this prevents from using fine quantization of the color space (although this may be unavoidable due to the insufficient quantization of color images). Finally, the extension to the case of more than two color images is not obvious.

As stated in the Introduction, the variational method proposed in this paper approaches the color histogram of the given images without pre-computing it and can be applied to more than two images.

\section{A VARIATIONAL APPROACH TO HISTOGRAM TRANSFER}

Our aim is to propose a variational formulation for midway histogram equalization. Assume that we are given two color images $\vec{U}: \Omega_{\vec{U}} \rightarrow \mathbb{R}^{3}$ and $\vec{V}: \Omega_{\vec{V}} \rightarrow \mathbb{R}^{3}$ where $\Omega_{\vec{U}}, \Omega_{\vec{V}}$ denote the corresponding image domains. We want to map the images $\vec{U}, \vec{V}$ onto new images $\vec{u}, \vec{v}$, respectively, so that $\vec{u}$ and $\vec{v}$ share, as much as possible, a common intermediate histogram between those of $\vec{U}$ and $\vec{V}$. Thus, the main ingredients of the proposed energy are the distance between the two color histograms of $\vec{u}$ and $\vec{v}$ and the constraints that impose $\vec{u}$ and $\vec{v}$ to display the same geometric information as $\vec{U}$ and $\vec{V}$, respectively.

In order to define such an energy, we need to discuss first the variational formulation of histogram transfer based on the $L^{2}$ distance between two histograms. In order to make our presentation more comprehensible, we shall discuss first the case of gray level images (which have one-dimensional histograms) and compute the first variation of the corresponding energy. This will clarify the differences between this functional and the transport approach to midway histogram equalization and will also explain why we need to impose color and geometric constraints. We will then consider the two-dimensional case, $N=2$, presenting the energy functional and its first variation. The rigorous computation details and the formulae of the color case, $N=3$, are quite cumbersome, for this reason we will discuss them in the Appendix VIII.

Let us first fix the notation that will be used throughout the paper. 


\section{A. Notation}

We assume that images are defined on a discrete rectangular domain, which we denote by $\Omega=\{1, \ldots, W\} \times\{1, \ldots, H\} \subset \mathbb{N}^{2}, W, H \geq 1$ being integers; $x=\left(x_{1}, x_{2}\right)$ denote the coordinates of an arbitrary pixel in $\Omega$. We assume that each image channel has a normalized dynamic range which we suppose to be $[0,1]$, so that images are described by vector functions $\vec{I}: \Omega \rightarrow[0,1]^{N}, \vec{I}(x)=\left(I_{1}(x), \ldots, I_{N}(x)\right)$. For color images, we have $N=3$ and $I_{k}(x)$ is the intensity level of the pixel $x \in \Omega$ in the chromatic channel $k \in\{R, G, B\}$. The histogram of the image $\vec{I}$ will be denoted by the density function $h_{\vec{I}}:[0,1]^{N} \mapsto[0,1]$, while $H_{\vec{I}}:[0,1]^{N} \mapsto[0,1]$ will represent the cumulative histogram of $\vec{I}$. Thus, if $\vec{\lambda}=\left(\lambda_{1}, \ldots, \lambda_{N}\right) \in[0,1]^{N}$ represents the generic $N$-dimensional intensity level, we have:

$$
H_{\vec{I}}(\vec{\lambda})=\int_{0}^{\lambda_{1}} \cdots \int_{0}^{\lambda_{N}} h_{\vec{I}}(\vec{\mu}) d \vec{\mu} .
$$

Note that the cumulative histogram can be equivalently defined from the image $\vec{I}$ as:

$$
H_{\vec{I}}(\vec{\lambda})=\frac{1}{|\Omega|} \mid\left\{x \in \Omega, \text { such that } I_{1}(x) \leq \lambda_{1}, \ldots, I_{N}(x) \leq \lambda_{N}\right\} \mid,
$$

where $\mid$ denotes the area operator.

B. The $L^{2}$ distance between the cumulative histogram of a gray-scale image and a reference one and its first variation

Let $I: \Omega \rightarrow[0,1]$ be a gray level image whose cumulative histogram is $H_{I}(\lambda)=\frac{1}{|\Omega|} \mid\{x \in$ $\Omega: I(x) \leq \lambda\} \mid, \lambda \in[0,1]$. Let $H_{\text {ref }}(\lambda)$ be a given reference cumulative histogram. In order to impose the cumulative histogram of $I$ to be close to $H_{\text {ref }}(\lambda)$ we propose to minimize the following energy:

$$
\mathcal{E}(I)=\frac{1}{2} \int_{0}^{1}\left(H_{I}(\lambda)-H_{\mathrm{ref}}(\lambda)\right)^{2} d \lambda .
$$

Obviously, the minimum would be attained when $H_{I}(\lambda)=H_{\text {ref }}(\lambda)$. This energy term will be later used together with other (attachment to data and shape-preserving) terms in order to construct an energy for intermediate histogram equalization of a given set of images.

Let us compute the first variation of the energy (5). For that, observe that we may write

$$
H_{I}(\lambda)=\frac{1}{|\Omega|} \sum_{x \in \Omega} \mathrm{h}_{\lambda}(I(x)),
$$


where

$$
\mathrm{h}_{\lambda}(r)=\chi_{[0, \lambda]}(r) \quad \lambda, r \geq 0,
$$

$\chi_{[0, \lambda]}$ being the characteristic function of the set $[0, \lambda]$. Hence, if $J: \Omega \rightarrow \mathbb{R}^{+}$denotes a perturbation, we have

$$
\begin{aligned}
\left.\frac{d}{d \epsilon}\right|_{\epsilon=0} H_{I+\epsilon J}(\lambda) & =\left.\frac{1}{|\Omega|} \frac{d}{d \epsilon}\right|_{\epsilon=0} \sum_{x \in \Omega} \mathrm{h}_{\lambda}(I(x)+\epsilon J(x)) \\
& =\left.\frac{1}{|\Omega|} \sum_{x \in \Omega} \frac{d}{d \epsilon}\right|_{\epsilon=0} \mathrm{~h}_{\lambda}(I(x)+\epsilon J(x)) \\
& =-\frac{1}{|\Omega|} \sum_{x \in \Omega} \delta(I(x)-\lambda) J(x) .
\end{aligned}
$$

Then

$$
\begin{aligned}
\left.\frac{d}{d \epsilon}\right|_{\epsilon=0} \mathcal{E}(I+\epsilon J) & =-\frac{1}{|\Omega|} \int_{0}^{1}\left(H_{I}(\lambda)-H_{\mathrm{ref}}(\lambda)\right) \sum_{x \in \Omega} \delta(I(x)-\lambda) J(x) d \lambda \\
& =-\frac{1}{|\Omega|} \sum_{x \in \Omega} \int_{0}^{1}\left(H_{I}(\lambda)-H_{\mathrm{ref}}(\lambda)\right) \delta(I(x)-\lambda) J(x) d \lambda \\
& =-\frac{1}{|\Omega|} \sum_{x \in \Omega}\left(H_{I}(I(x))-H_{\mathrm{ref}}(I(x))\right) J(x) .
\end{aligned}
$$

That is

$$
\nabla_{I} \mathcal{E}(I)=-\frac{1}{|\Omega|}\left(H_{I}(I(x))-H_{\mathrm{ref}}(I(x))\right) .
$$

Remark 3.1: We may use a gradient descent algorithm to minimize the energy (5). Initializing with $I^{0}=I$, for some positive evolution parameter step $\tau$, the process is described by the equation

$$
I^{k+1}=I^{k}-\tau \nabla_{I^{k}} \mathcal{E}\left(I^{k}\right) .
$$

Remark 3.2: If $H_{1}, H_{2}$ are two given reference cumulative histograms, the energy

$$
\mathcal{E}_{1,2}(I):=\frac{1}{2} \int_{0}^{1}\left(H_{I}(\lambda)-H_{1}(\lambda)\right)^{2} d \lambda+\frac{1}{2} \int_{0}^{1}\left(H_{I}(\lambda)-H_{2}(\lambda)\right)^{2} d \lambda .
$$

expresses the distance of $H_{I}$ to $H_{1}$ and $H_{2}$. Using (10), the Euler-Lagrange equation $\nabla_{I} \mathcal{E}_{1,2}(I)=$ 0 can be written as

$$
H_{I}(I(x))=\frac{H_{1}(I(x))+H_{2}(I(x))}{2} .
$$

As we observed in Section II, this is not a suitable choice for intermediate histogram equalization since it may generate new unwanted structures (level lines) in the image. Thus, this energy term will be constrained in order to keep the geometry of the original image. Before going into details about this constraining, let us extend the above computations to the vector case. 
C. The $L^{2}$ distance between the cumulative histogram of a color image and a reference one and its first variation

Let us consider the case of vector valued images $\vec{I}: \Omega \rightarrow[0,1]^{N}$. To simplify the presentation, let us study here the case $N=2$, the case of color images with $N=3$ will be considered in Appendix VIII. Thus, if $\vec{I}=\left(I_{1}, I_{2}\right)$ and $\vec{\lambda}=\left(\lambda_{1}, \lambda_{2}\right) \in[0,1]^{2}$, the cumulative histogram of $I$ is defined by $H_{\vec{I}}(\vec{\lambda})=\frac{1}{|\Omega|}\left|\left\{x: I_{1}(x) \leq \lambda_{1}, I_{2}(x) \leq \lambda_{2}\right\}\right|$. Let $H_{\text {ref }}(\vec{\lambda})$ be a reference cumulative histogram. We then define the transfer energy:

$$
\mathcal{E}(\vec{I})=\frac{1}{2} \int_{0}^{1} \int_{0}^{1}\left(H_{\vec{I}}(\vec{\lambda})-H_{\text {ref }}(\vec{\lambda})\right)^{2} d \vec{\lambda} .
$$

If $\mathcal{H}^{1}, \mathcal{H}^{2}$ are defined by

$$
\mathcal{H}^{1}\left(i_{1}, i_{2}\right) \equiv \int_{0}^{i_{2}} H\left(i_{1}, \lambda_{2}\right) d \lambda_{2}, \quad \mathcal{H}^{2}\left(i_{1}, i_{2}\right) \equiv \int_{0}^{i_{1}} H\left(\lambda_{1}, i_{2}\right) d \lambda_{1},
$$

then, as it will be proven in Appendix VIII, the first variations of $\mathcal{E}(\vec{I})$ with respect to $I_{1}, I_{2}$ are

$$
\begin{aligned}
\nabla_{I_{1}} \mathcal{E}(\vec{I}) & =-\frac{1}{|\Omega|}\left[\mathcal{H}_{\vec{I}}^{1}\left(I_{1}(x), 1\right)-\mathcal{H}_{\mathrm{ref}}^{1}\left(I_{1}(x), 1\right)\right] \\
& +\frac{1}{|\Omega|}\left[\mathcal{H}_{\vec{I}}^{1}\left(I_{1}(x), I_{2}(x)\right)-\mathcal{H}_{\mathrm{ref}}^{1}\left(I_{1}(x), I_{2}(x)\right)\right] \\
\nabla_{I_{2}} \mathcal{E}(\vec{I}) & =-\frac{1}{|\Omega|}\left[\mathcal{H}_{\vec{I}}^{2}\left(1, I_{2}(x)\right)-\mathcal{H}_{\mathrm{ref}}^{2}\left(1, I_{2}(x)\right)\right] \\
& +\frac{1}{|\Omega|}\left[\mathcal{H}_{\vec{I}}^{2}\left(I_{1}(x), I_{2}(x)\right)-\mathcal{H}_{\mathrm{ref}}^{2}\left(I_{1}(x), I_{2}(x)\right)\right] .
\end{aligned}
$$

\section{Symmetrization of the cumulation of histogram}

Let us mention that, by definition of cumulative histogram, the process presented above presents an important unwanted limitation that can be simply explained in the case $N=1$. Indeed, note that for any grey image $I: \Omega \mapsto[0,1]$, we have $H_{I}(1)=\frac{1}{|\Omega|}|\{x: I(x) \leq 1\}|=1$. As a consequence, when computing the gradient value of white pixels of color value 1 , we will always find $H_{I}(1)-H_{\text {ref }}(1)=1-1=0$, so the white pixels can never change their intensity value. To remove this limitation, we need to consider $H^{*}$, the histogram defined as $H_{I}^{*}(\lambda)=|\{x: I(x) \geq \lambda\}|, \lambda \in[0,1]$. The energy (5) should then be completed with the term $\frac{1}{2} \int_{0}^{1}\left(H_{I}^{*}(\lambda)-H_{\mathrm{ref}}^{*}(\lambda)\right)^{2} d \lambda$, so that the symmetrized energy will be

$$
\mathcal{E}(I)=\frac{1}{2} \int_{0}^{1}\left[\left(H_{I}(\lambda)-H_{\mathrm{ref}}(\lambda)\right)^{2}+\left(H_{I}^{*}(\lambda)-H_{\mathrm{ref}}^{*}(\lambda)\right)^{2}\right] d \lambda,
$$


that will allow the white pixels to move their value. Obviously, by symmetry, this new term will not affect the black pixels, as $H_{I}^{*}(0)=1$ for any image $I: \Omega \rightarrow[0,1]$. For the $N$-dimensional case, $N>1$, we have

$$
\mathcal{E}(\vec{I})=\frac{1}{2} \underbrace{\int_{0}^{1} \cdots \int_{0}^{1}}_{N \text { times }}\left[\left(H_{\vec{I}}(\vec{\lambda})-H_{\mathrm{ref}}(\vec{\lambda})\right)^{2}+\left(H_{\vec{I}}^{*}(\vec{\lambda})-H_{\mathrm{ref}}^{*}(\vec{\lambda})\right)^{2}\right] d \vec{\lambda},
$$

It is clear that the gradient formulae previously written still hold for the symmetrized energy, modulo the introduction of a symmetric term, or terms (for the higher dimensional case), in $H^{*}$.

\section{INTERMEDIATE HISTOGRAM EQUALIZATION OF A SET OF IMAGES}

As reviewed in section II, histogram equalization is mainly addressed from the point of view of mass transfer [6], [13], [15], where the unknown is the transfer function $\varphi:[0,1]^{N} \mapsto[0,1]^{N}$ mapping a variable histogram to a reference one. Considering the problem of equalizing (at least) two different images with respect to their midway histogram, these methods require an $a$ priori computation of the midway histogram. The purpose of this section is to show how can we evolve simultaneously different images so that their histograms get naturally as close as possible to an unspecified intermediate histogram.

\section{A. An energy term to approach the histogram of two images}

Suppose that we are given two color images $\vec{U}: \Omega_{\vec{U}} \rightarrow[0,1]^{3}$ and $\vec{V}: \Omega_{\vec{V}} \rightarrow[0,1]^{3}$, $\vec{U}=\left(U_{1}, U_{2}, U_{3}\right), \vec{V}=\left(V_{1}, V_{2}, V_{3}\right)$, and we want to map them into two images with a common histogram as much as this is possible. We can then try to construct two color images $\vec{u}=$ $\left(u_{1}, u_{2}, u_{3}\right)$ and $\vec{v}=\left(v_{1}, v_{2}, v_{3}\right)$ related to $\vec{U}, \vec{V}$, respectively, and defined on the spatial domains $\Omega_{\vec{U}}$ and $\Omega_{\vec{V}}$, so that they minimize the histogram-based energy $\mathcal{E}_{H}$ :

$$
\mathcal{E}_{H}(\vec{u}, \vec{v})=\frac{1}{2} \int_{0}^{1} \int_{0}^{1} \int_{0}^{1}\left(H_{\vec{u}}(\vec{\lambda})-H_{\vec{v}}(\vec{\lambda})\right)^{2} d \vec{\lambda}+\frac{1}{2} \int_{0}^{1} \int_{0}^{1} \int_{0}^{1}\left(H_{\vec{u}}^{*}(\vec{\lambda})-H_{\vec{v}}^{*}(\vec{\lambda})\right)^{2} d \vec{\lambda}
$$

That is, $\vec{u}, \vec{v}$ should have similar histograms and be relatively close to the original images $\vec{U}, \vec{V}$.

Note that, even if the final histograms of $\vec{u}$ and $\vec{v}$ are equal, we cannot guarantee that this is the true midway histogram of $\vec{U}$ and $\vec{V}$ (in the sense described in Section II). If we consider for instance two unimodal images $\vec{U}=\lambda_{0}$ and $\vec{V}=\lambda_{1}$, all pixels of each image are initially equal and they will always share a common value all along the minimization process (since in this 
case, the gradient (10) is the same for the whole image). Then, we could obtain any unimodal image $\vec{u}=\vec{v}=s, s \in[0,1]$, as a minimum of energy (20), while we would prefer finding the particular value $s \equiv \frac{\lambda_{0}+\lambda_{1}}{2}$. This value can be obtained if we add a data attachment term to enforce that $\vec{u}, \vec{v}$ are, respectively, close to $\vec{U}, \vec{V}$. Thus, we propose to complement the energy term (20) with data attachment terms constraining the color and the geometric information of the images $\vec{u}, \vec{v}$ to be close to the one in $\vec{U}, \vec{V}$.

\section{B. Attachment to data and to the image geometry}

Contrary to the methods based on histogram transfer, as our variables are the images, we can directly add some color and geometrical restrictions on the image evolution.

a) Constraining the colors: In order to enforce $\vec{u}$ and $\vec{v}$ to remain as close as possible to the original images $\vec{U}$ and $\vec{V}$ we define the following attachment to data energy term $\mathcal{E}_{D}$ :

$$
\mathcal{E}_{D}(\vec{u}, \vec{v})=\frac{\alpha_{\vec{U}}}{2} \sum_{x \in \Omega_{\vec{U}}}\|\vec{U}(x)-\vec{u}(x)\|^{2}+\frac{\alpha_{\vec{V}}}{2} \sum_{x \in \Omega_{\vec{V}}}\|\vec{V}(x)-\vec{v}(x)\|^{2},
$$

where $\alpha_{\vec{U}}$ and $\alpha_{\vec{V}}$ are positive real parameters. With this new term, by setting $\alpha_{\vec{U}}=\alpha_{\vec{V}}$, we can constrain the final images $\vec{u}$ and $\vec{v}$ to have a similar distance to their original values $\vec{U}$ and $\vec{V}$.

Let us go back to the unimodal toy example $\vec{U}=\lambda_{0}$ and $\vec{V}=\lambda_{1}$ and consider the energy $\mathcal{E}_{H D}=\mathcal{E}_{H}+\mathcal{E}_{D}$, with $\alpha_{\vec{U}}=\alpha_{\vec{V}}=\epsilon>0$. In this case both solutions $\vec{u}, \vec{v}$ are unimodal, say $\vec{u}=\lambda_{0}, \vec{v}=\lambda_{1}$ with $\lambda_{0}<\lambda_{1}$. Then we can write the energy $\mathcal{E}_{H D}$ in terms of $\lambda_{0}, \lambda_{1}$ and check that when $\epsilon>0$ is small enough (to constrain the histogram term $\mathcal{E}_{H}$ to be preponderant in $\mathcal{E}_{H D}$ ) the minimum of $\mathcal{E}_{H D}$ is reached for the unimodal image $\vec{u}=\vec{v}=\frac{\lambda_{0}+\lambda_{1}}{2}$ whose histogram corresponds to the midway one ${ }^{1}$.

In real applications, the energy $\mathcal{E}_{H}$ is obviously never zero, so we do not reach the true midway histogram. Nevertheless, from our experiments, we can assert that the histogram and data energies (20) and (21) allow the histograms of images $\vec{u}$ and $\vec{v}$ to converge naturally to a histogram in a neighborhood of the midway histogram of images $\vec{U}$ and $\vec{V}$.

\footnotetext{
${ }^{1}$ This would not be the case by considering a $L^{1}$ attachment to data term $\mathcal{E}_{D}(\vec{u}, \vec{v})=\alpha_{U}\|U-u\|_{1}+\alpha_{V}\|V-v\|_{1}$. Indeed, the energy $\mathcal{E}_{H, D}$ would have the same value for any unimodal image $\vec{u}=\vec{v}=s, s \in\left[\lambda_{0}, \lambda_{1}\right]$.
} 
b) Constraining the image geometry: We can constrain the shapes of $\vec{u}$ and $\vec{v}$ to be those of the given images $\vec{U}$ and $\vec{V}$. To describe the shapes of $U_{i}, V_{i}$ we use the normals to its level lines, that is, we use the vector fields $\theta\left(U_{i}\right)=\frac{\nabla U_{i}}{\left|\nabla U_{i}\right|}, \theta\left(V_{i}\right)=\frac{\nabla V_{i}}{\left|\nabla V_{i}\right|}$ [17] (the quotient must be set to 0 when the denominator is 0 ) where $\nabla$ denotes here a discrete gradient operator (see Section $\mathrm{V}-\mathrm{B}$ ). Then we say that the scalar image $u_{i}$ shares the same shapes as $U_{i}$ (in some sense, the same level lines) if $\theta\left(U_{i}\right) \bullet \theta\left(u_{i}\right)=1$, i.e. when $\theta\left(U_{i}\right) \bullet \nabla u_{i}=\left|\nabla u_{i}\right|$ (see [17] for more details).

In order to constrain the shapes of $\vec{u}$ and $\vec{v}$ to be those of $\vec{U}$ and $\vec{V}$, respectively, we consider (together with the histogram and color terms) the following shape preserving energy $\mathcal{E}_{S}$

$$
\mathcal{E}_{S}(\vec{u}, \vec{v})=\beta_{\vec{U}} \sum_{i=1}^{3} \sum_{\Omega_{\vec{U}}}\left(\left|\nabla u_{i}\right|-\theta(U)_{i} \bullet \nabla u_{i}\right)+\beta_{\vec{V}} \sum_{i=1}^{3} \sum_{\Omega_{\vec{V}}}\left(\left|\nabla v_{i}\right|-\theta(V)_{i} \bullet \nabla v_{i}\right),
$$

where $\beta_{\vec{U}}$ and $\beta_{\vec{V}}$ are positive parameters. Imposing the channels of two color images $\vec{U}$ and $\vec{u}$ to share the same shapes (or level lines) is not a strong constraint on the color distributions.

Remark 4.1: A related approach. In the case of histogram transfer by estimation of the transfer function $\varphi:[0,1]^{3} \rightarrow[0,1]^{3}$, an analogous local shape preservation is sometimes applied as a post-processing. For instance, in [6], the authors tackle the problem of transferring the histogram $h_{\vec{V}}$ of an image $\vec{V}$ to the histogram $h_{\vec{U}}$ of image $\vec{U}$. First, they compute the transfer function $\varphi$ so that $h_{\varphi \circ \vec{U}} \simeq h_{\vec{V}}$ and then look for an image $\vec{u}$ sharing the shapes of the original image $\vec{U}$ and whose color histogram is $h_{\varphi \circ \vec{U}}$ by solving:

$$
\min _{\vec{u}} \sum_{i=1}^{3} \sum_{x \in \Omega_{\vec{U}}}\left|\nabla U_{i}(x)-\nabla u_{i}(x)\right|+\sum_{x \in \Omega_{\vec{U}}}|\vec{u}(x)-\varphi(\vec{U}(x))| .
$$

We note that $\varphi(\vec{U}(x))$ is known since the transfer function is previously computed. Different strategies for that can be found in [6], [13]. For instance, in [13], the following Monge's optimal transportation problem is solved:

$$
\min _{\varphi} \int_{0}^{1} \int_{0}^{1} \int_{0}^{1}\|\varphi(\vec{\lambda})-\vec{\lambda}\|^{2} h_{\vec{U}}(\vec{\lambda}) d \vec{\lambda}
$$

where the possible transfer maps $\varphi$ are restricted to be linear. The optimal transfer map minimizes the displacement cost of colors when mapping the histogram of $\vec{U}$ to $h_{\vec{V}}$.

\section{A model for intermediate histogram equalization of a set of images}

Combining the histogram energy (20) with the color and geometry preserving terms of Section IV-B, we finally get the following energy functional for equalizing the color of two images $\vec{U}$ 
and $\vec{V}$ :

$$
\mathcal{E}_{\vec{U}, \vec{V}}(\vec{u}, \vec{v})=\mathcal{E}_{H}(\vec{u}, \vec{v})+\mathcal{E}_{D}(\vec{u}, \vec{v})+\mathcal{E}_{S}(\vec{u}, \vec{v}) .
$$

The energy can be extended to deal with the equalization of more than two images. Let us consider $\vec{U}^{k}, k=1, \ldots, m$, a set of color images. We can then use the energy (25) and define the following problem

$$
\min _{\vec{u}_{1}, \ldots, \vec{u}_{m}} \sum_{1 \leq k<l \leq m} \mathcal{E}_{\vec{U}^{k}, \vec{U}^{l}}\left(\vec{u}_{k}, \vec{u}_{l}\right),
$$

in order to normalize the color of the set of images $\vec{U}^{k}$.

\section{DESCRIPTION OF AN ALGORITHM TO MINIMIZE $\mathcal{E}_{\vec{U}, \vec{V}}$}

In this Section we describe the algorithm we used to minimize (25).

\section{A. A primal-dual algorithm}

Since the shape preserving term (22) includes a total variation regularization, we minimize $\mathcal{E}$ through a primal-dual proximal point algorithm. Let us detail this process for the minimization of $\mathcal{E}$ with respect to $\vec{u}$ when $\vec{v}$ is fixed. Discarding the terms involving only $\vec{v}$, we have

$$
\begin{aligned}
& \min _{\vec{u}} \mathcal{E}_{H}(\vec{u}, \vec{v})+\frac{\alpha_{\vec{U}}}{2} \sum_{x \in \Omega_{\vec{U}}}\|\vec{U}(x)-\vec{u}(x)\|^{2}+\beta_{\vec{U}} \sum_{i=1}^{3} \sum_{\Omega}\left(\left|\nabla u_{i}\right|-\theta(U)_{i} \cdot \nabla u_{i}\right) \\
= & \min _{\vec{u}} \max _{\vec{\sigma} \in S} \mathcal{E}_{H}(\vec{u}, \vec{v})+\frac{\alpha_{\vec{U}}}{2} \sum_{x \in \Omega_{\vec{U}}}\|\vec{U}(x)-\vec{u}(x)\|^{2}+\beta_{\vec{U}} \sum_{i=1}^{3} \sum_{\Omega}\left(\sigma_{i} \cdot \nabla u_{i}-\theta(U)_{i} \cdot \nabla u_{i}\right) \\
= & \min _{\vec{u}} \max _{\vec{\sigma} \in S} \mathcal{E}_{H}(\vec{u}, \vec{v})+\frac{\alpha_{\vec{U}}}{2} \sum_{x \in \Omega_{\vec{U}}}\|\vec{U}(x)-\vec{u}(x)\|^{2}-\beta_{\vec{U}} \sum_{i=1}^{3} \sum_{\Omega} \operatorname{div}\left(\sigma_{i}-\theta(U)_{i}\right) u_{i},
\end{aligned}
$$

where $S=\left\{\vec{\sigma}=\left(\sigma_{1}, \sigma_{2}, \sigma_{3}\right)\right.$, with $\sigma_{i}: \Omega_{\vec{U}} \times \Omega_{\vec{U}} \rightarrow \mathbb{R}^{2}$ and $\left.\left|\sigma_{i}\right| \leq 1\right\}$.

Starting from initial values $\vec{u}^{0}$ and $\vec{\sigma}^{0} \in S$ and given two positive time steps $\tau$ and $\tau_{\sigma}$, the primal-dual proximal point algorithm consists of a gradient ascent method for the dual variable $\sigma$ and a gradient descent for the variable $\vec{u}$. The dual step is then

$$
\sigma_{i}^{k+1}=\sigma_{i}^{k}+\tau_{\sigma} \beta_{\vec{U}} \nabla u_{i}^{k} .
$$


while the primal step is

$$
\begin{aligned}
u_{i}^{k+1} & =u_{i}^{k}-\tau \nabla_{u} \mathcal{E}_{\vec{U}, \vec{V}}\left(\vec{u}^{k}, \vec{v}^{k}\right) \\
& =u_{i}^{k}-\tau\left(\nabla_{u} \mathcal{E}_{H}\left(\vec{u}^{k}, \vec{v}^{k}\right)+\alpha_{\vec{U}}\left(u_{i}^{k}-U_{i}\right)-\beta_{\vec{U}} \operatorname{div}\left(\sigma_{i}^{k}-\theta(U)_{i}\right)\right)
\end{aligned}
$$

where the gradient $\nabla_{u_{i}^{k}} \mathcal{E}_{H}\left(\vec{u}^{k}, \vec{v}^{k}\right)$ can be computed as in the Appendix. At the end of each step, the dual vector variable $\sigma_{i}^{k+1}$ is projected into the unitary ball $S$, while the variable $u_{i}^{k+1}$ is projected into $[0,1]$.

Similar equations can be written for the variables $\vec{v}$.

\section{B. The discrete gradient and divergence}

Let us first give the definition of the discrete gradient and divergence operators. Assume that the discrete spatial domain is $\Omega:=\{1, \ldots, W\} \times\{1, \ldots, H\}$. A scalar image is thus a vector in $X:=\mathbb{R}^{W \times H}$. If $u \in X$, the discrete gradient is a vector in $X \times X$ given by

$$
\nabla u=\left[\begin{array}{l}
u_{x}^{+} \\
u_{y}^{+}
\end{array}\right]
$$

where

$$
u_{x}^{+}(i, j)= \begin{cases}u(i+1, j)-u(i, j) & \text { if } i<W \\ 0 & \text { if } i=W\end{cases}
$$

and

$$
u_{y}^{+}(i, j)= \begin{cases}u(i, j+1)-u(i, j) & \text { if } j<H \\ 0 & \text { if } j=H\end{cases}
$$

The discrete divergence operator is determined by the relation $\langle\operatorname{div} \sigma, u\rangle_{X}=-\langle\sigma, \nabla u\rangle_{X \times X}$ for all $u \in X, \sigma=\left(\sigma^{1}, \sigma^{2}\right) \in X \times X$, where $\langle\cdot, \cdot\rangle_{X},\langle\cdot, \cdot\rangle_{X \times X}$ denote the scalar products in $X$, $X \times X$, respectively. Thus, $\operatorname{div} \sigma=\left(\sigma^{1}\right)_{x}^{-}+\left(\sigma^{2}\right)_{y}^{-}$, where

$$
\left(\sigma^{1}\right)_{x}^{-}(i, j)= \begin{cases}\sigma^{1}(i, j) & \text { if } i=1, \\ \sigma^{1}(i, j)-\sigma^{1}(i-1, j) & \text { if } 1<i<W, \\ -\sigma^{1}(i-1, j) & \text { if } i=W\end{cases}
$$

and

$$
\left(\sigma^{2}\right)_{y}^{-}(i, j)= \begin{cases}\sigma^{2}(i, j) & \text { if } j=1, \\ \sigma^{2}(i, j)-\sigma^{2}(i, j-1) & \text { if } 1<j<H \\ -\sigma^{2}(i, j-1) & \text { if } j=H .\end{cases}
$$


Finally, let us underline that the histograms are discretized in bins. In practice, we used $M$ bins for each channel, so that the dimension of the histograms is $M^{3}$. The value of the histogram $H_{I}$ for the color $\left(\lambda_{1}, \lambda_{2}, \lambda_{3}\right)$ is thus $H_{I}\left(\left[\lambda_{1} M\right],\left[\lambda_{2} M\right],\left[\lambda_{3} M\right]\right)$, where $[\cdot]$ denotes the integer part of its argument.

\section{EXPERIMENTS}

In all our experiments, we used the same set of parameters. The histogram is discretized into $M=64$ bins. Concerning the model parameters, we used $\alpha_{\vec{U}}=\alpha_{\vec{V}}=0.1$ for the data term and $\beta_{\vec{U}}=\beta_{\vec{V}}=0.1$ for the shape preserving term. The time steps for the primal-dual proximal point algorithm are fixed as $\tau=\tau_{\sigma}=10^{-4}$.

\section{A. Histogram transfer}

We first apply our process with $\vec{v}=\vec{V}$ being fixed, so that only the image $\vec{u}$ can change its values. In this case, we recover the same problem as the one considered in [13]. As the code of [13] is available on the web ${ }^{2}$, we have compared this method with ours in terms of the distance between histograms. If $h_{\vec{u}}$ and $h_{\vec{v}}$ are the histograms of images $\vec{u}$ and $\vec{v}$, respectively, both methods are compared by means of the quantity

$$
\int_{0}^{1} \int_{0}^{1} \int_{0}^{1}\left(h_{\vec{u}}(\vec{\lambda})-h_{\vec{v}}(\vec{\lambda})\right)^{2} d \lambda
$$

computed for the images $\vec{u}$ obtained using both our method and the one in [13].

In [13], the authors show experiments on color grading between very different images. We think that this kind of experiments is not well adapted to compare different methods, as there is no ground truth available. As a consequence, we used a data set composed of pairs of images showing the same painting which are taken by unknown cameras and with different illuminations. We considered paintings, as they involve strong color contrast, and are quite close to the examples of color grading shown in [13]. This data set is thus favorable to the application of [13]. The original and final images obtained by both methods, the one in [13] and ours, on the paintings are shown in Figures 1 and 2. As illustrated in table I, both approaches give similar errors in the sense of (35), the method of [13] being slightly better in general. Both methods, nevertheless, give good results in terms of visual quality.

\footnotetext{
${ }^{2}$ http://www.mee.tcd.ie/ sigmedia/Research/ColourGrading
} 


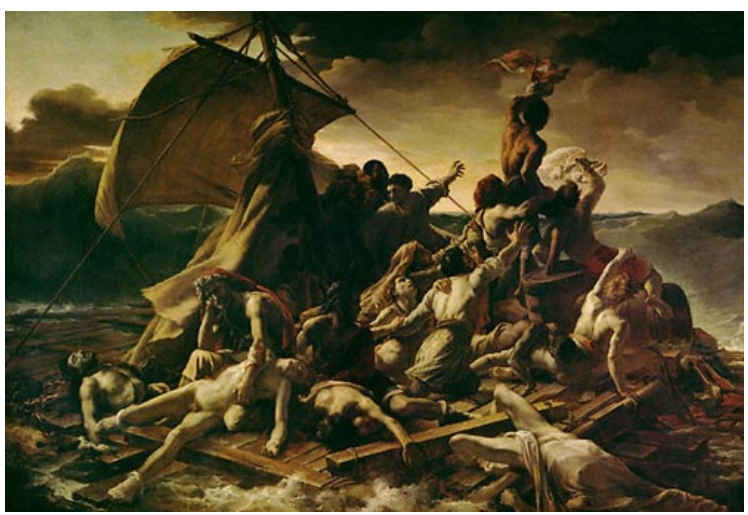

Reference image $\vec{V}$

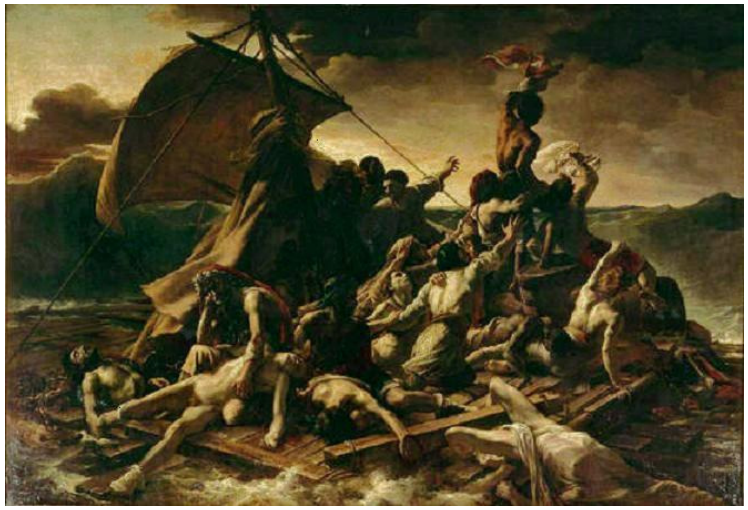

Result $\vec{u}$ with [13]

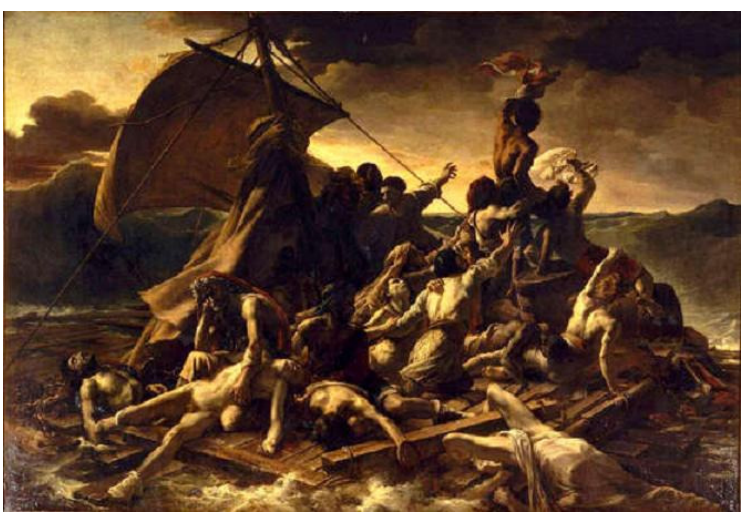

Original image $\vec{U}$

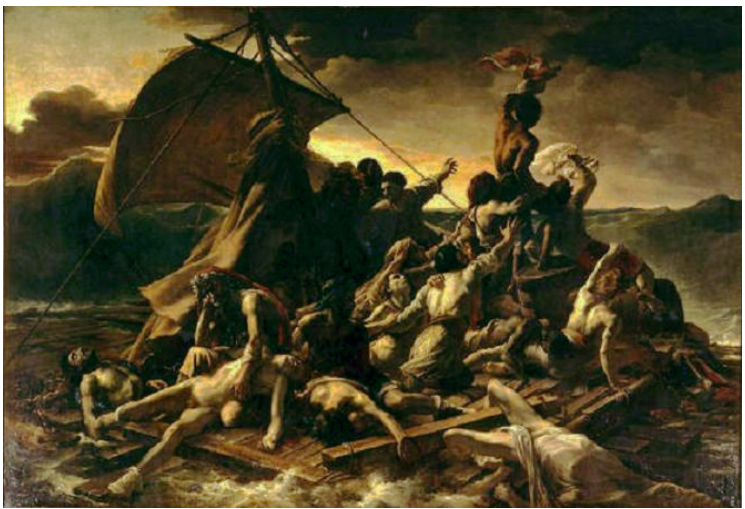

Result $\vec{u}$ with our method

Fig. 1. Comparison between our method and the one of [13] for the painting of Géricault: The Raft of the Medusa.

\begin{tabular}{|l||c|c|c|}
\hline Data & Initial error & Final error with [13] & Final error with our method \\
\hline \hline Medusa & 0.001576 & 0.000149 & 0.000215 \\
\hline Joconde & 0.010981 & 0.001281 & 0.002050 \\
\hline Tentation & 0.001766 & 0.000223 & 0.000415 \\
\hline Rising Sun & 0.001134 & 0.000188 & 0.000115 \\
\hline Cypress & 0.000163 & 0.000025 & 0.000077 \\
\hline
\end{tabular}

TABLE I

QUANTITATIVE COMPARISON BETWEEN OUR METHOD AND THE ONE OF [13] WITH CRITERIUM (35).

\section{B. Intermediate histogram equalization of a set of images}

Let us consider $\vec{U}^{k}, k=1, \ldots, m$, a set of color images representing the same scene with different orientations or illuminations. We solve the problem (26) in order to normalize the color of the set of images $\vec{U}^{k}$. As already commented, we underline that, to our knowledge, there is no method in the literature that allows realizing such a process without defining a midway histogram beforehand. In [3], [12], when $m=2$, the problem has been considered from the point of view 

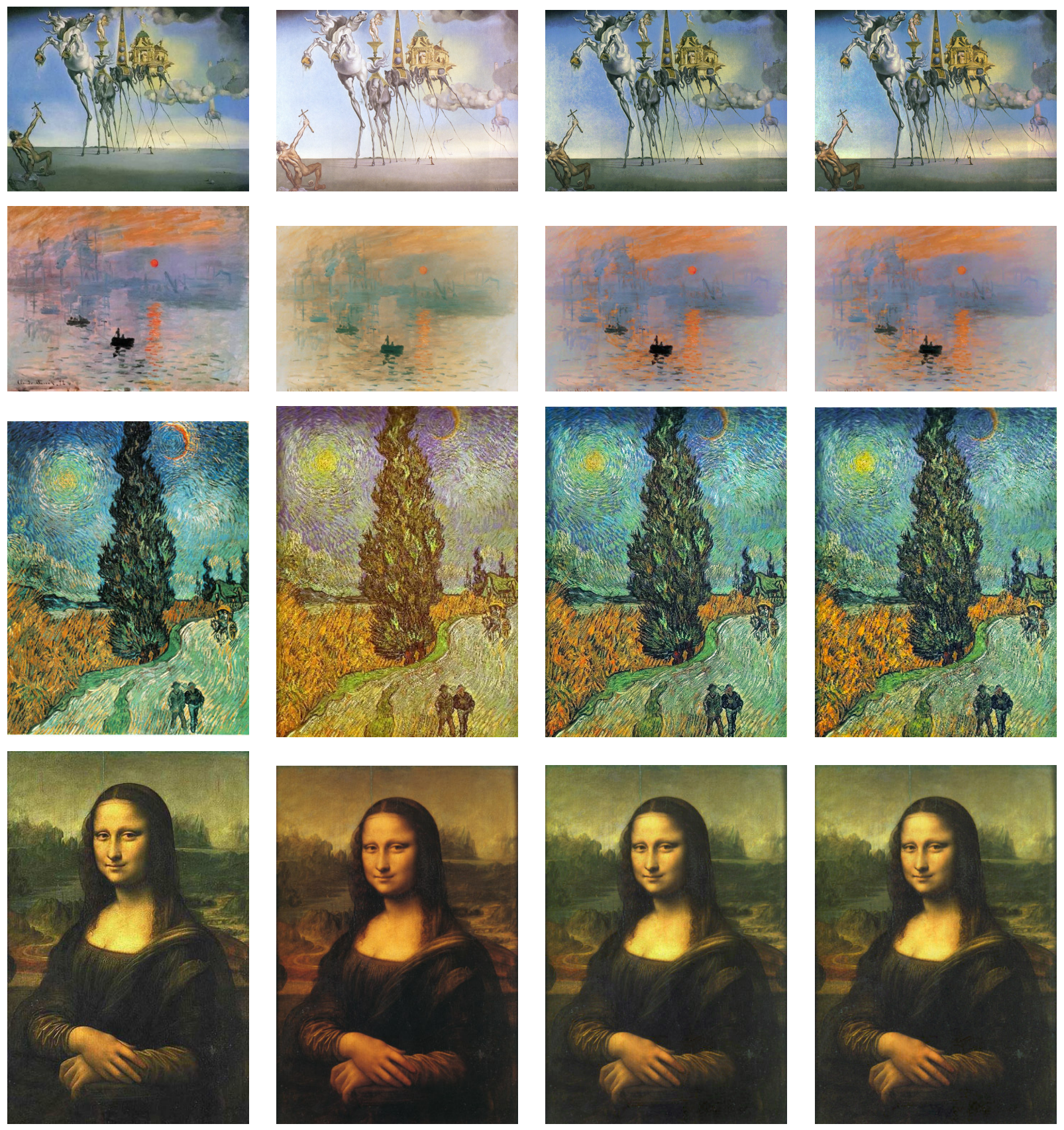

Reference image $\vec{V}$

Original image $\vec{U}$

Result $\vec{u}$ with [13]

Result $\vec{u}$ with our method

Fig. 2. Comparison between our method and the one of [13] for our data set. First row: Dali, Tentation. Second row: Monet, Impression Rising Sun. Third row: Van Gogh: Cypress against a starry sky. Fourth row: Da Vinci, Joconde.

of Monge-Kantorovich mass transportation theory leading to a linear programming problem for the computation of the transport plan transferring both histograms. Transport plans permit to 
split the mass of a bin of one of the histograms and distribute it between several bins in the other one. In [13], assuming that the histograms of two given color images can be approximated by multivariate Gaussian distributions, the Monge-Kantorovich formulation is restricted to find a linear map that approximates the transfer of histograms. The color grading method proposed in [6] is based on the computation of a transfer map and could be adapted to compute a midway histogram equalization (see [15]) in the case of two images. The transfer map is computed by iteratively equalizing all principal axis marginals computed for every possible rotation of the histogram, until convergence. Then the method is refined by a filtering process to reduce possible grain artifacts [6]. As mentioned in Section IV-B, this process is related to our geometry attachment term. Thus our method, although different in spirit, has common points with [6]. On the other hand, it is more adapted to the consideration of more than two images.

In Figures 3, 4 and 5 we show some examples of intermediate histogram equalization for sets of three images. 


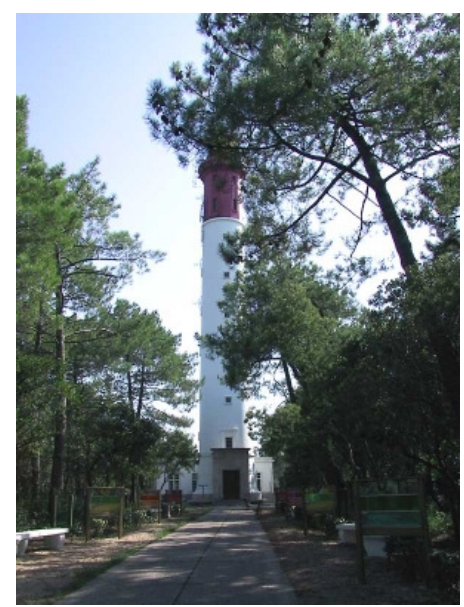

$\vec{U}^{1}$

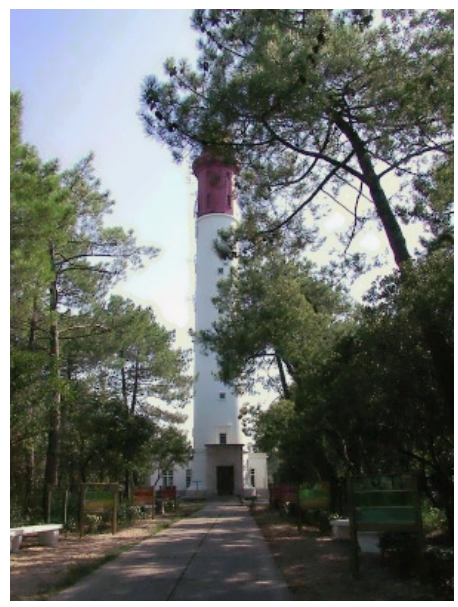

$\vec{u}^{1}$

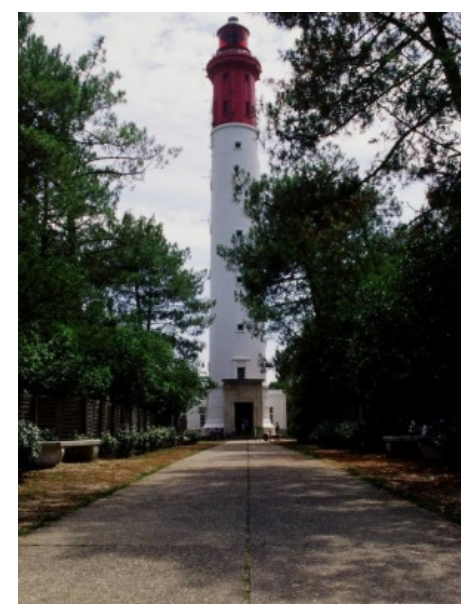

$\vec{U}^{2}$

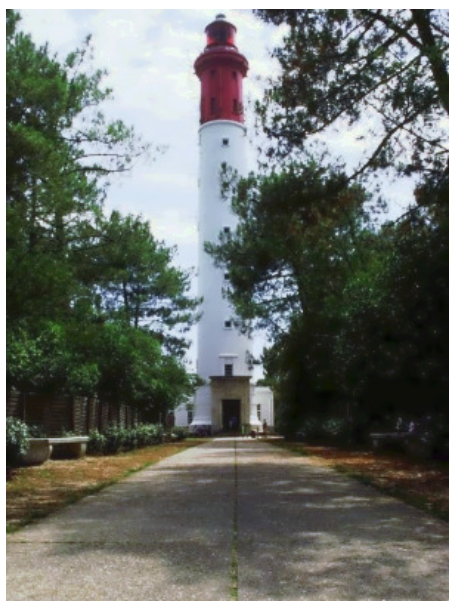

$\vec{u}^{2}$

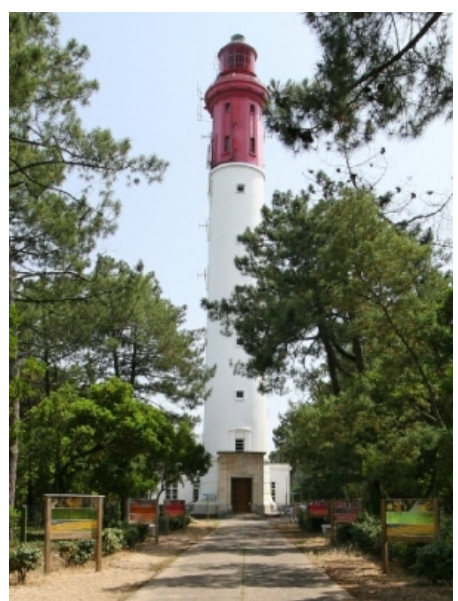

$\vec{U}^{3}$

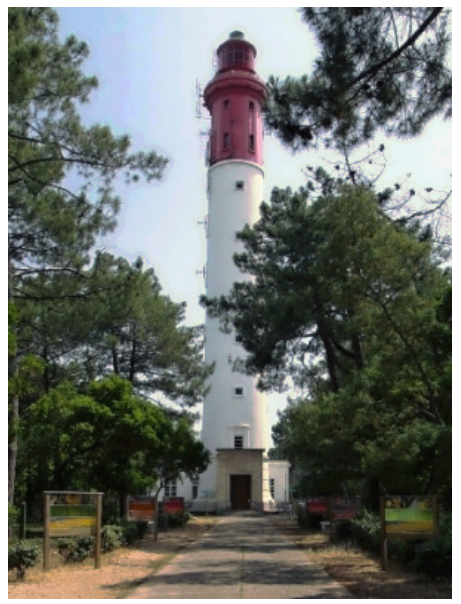

$\vec{u}^{3}$

Fig. 3. Lighthouse sequence. First line: Original images. Second line: Final images.

\section{CONCLUSIONS AND PERSPECTIVES}

We have proposed in this paper a variational model for intermediate color histogram equalization of two or more color images. Given a set of images, we transform them by minimizing an energy functional which is composed by three different terms: on one side we have a histogram transport term that tends to approach the cumulative histograms of the original images; on the other side we have two conservative terms that tend to maintain unaltered color and image geometry. The balance between these two opposite actions is vital for our purposes: the original images evolve in order to approach a common intermediate histogram that does not need to be specified in advance. Up to authors' knowledge, this is a novel property in the literature 


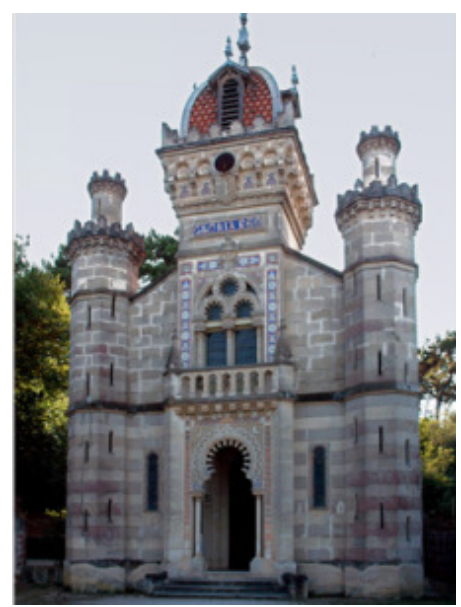

$\vec{U}^{1}$

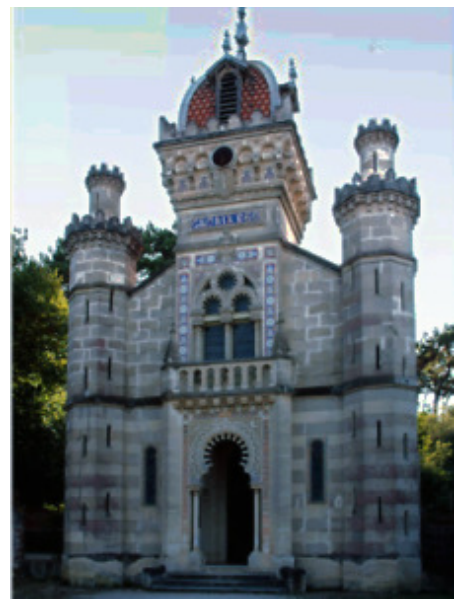

$\vec{u}^{1}$

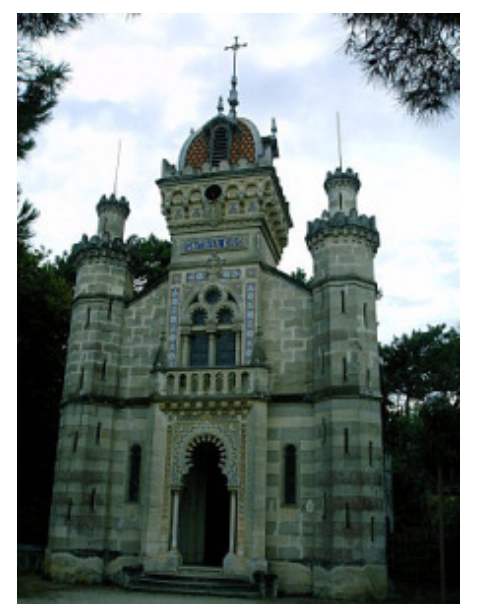

$\vec{U}^{2}$

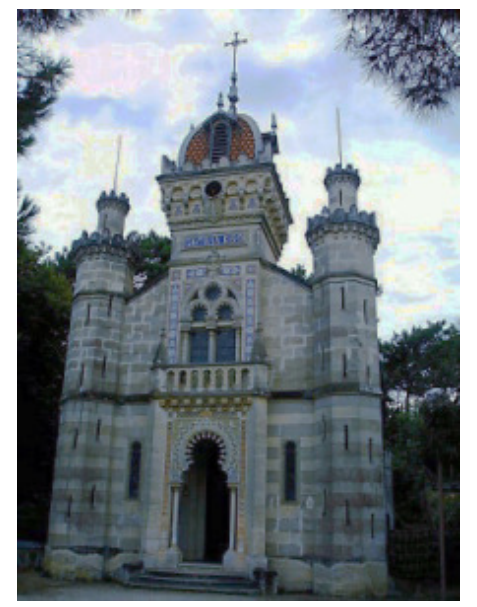

$\vec{u}^{2}$
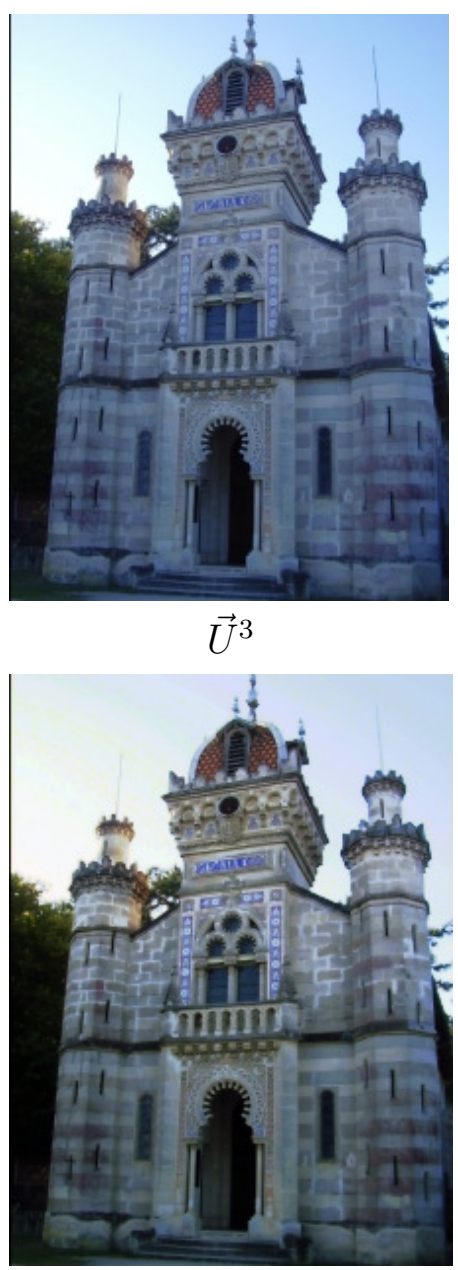

$\vec{u}^{3}$

Fig. 4. Chapel sequence. First line: Original images. Second line: Final images.

on the present subject. We have developed a primal-dual algorithm to minimize the proposed energy functional and shown that its results compare well with the state of the art. The flexibility provided by the variational framework enables to think about various refinements of the proposed model: for instance, one could introduce a local color enhancement term to enforce the action of the algorithm discussed in this paper on images affected by color cast. This condition is quite common, in fact most pictures are taken under illuminant conditions that can be very different from the standard D65 'white light', thus a color cast is very likely to be present in the corresponding image. 


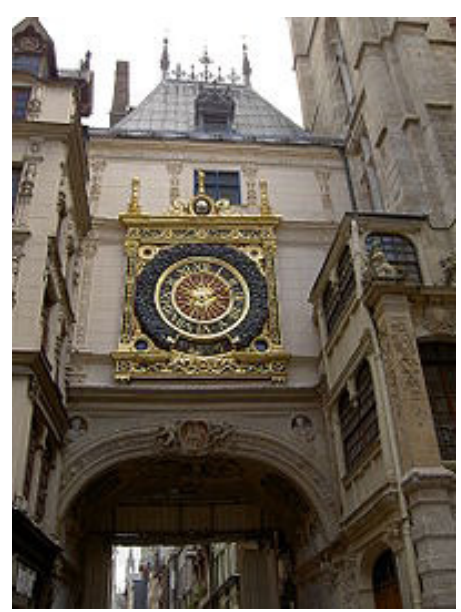

$\vec{U}^{1}$

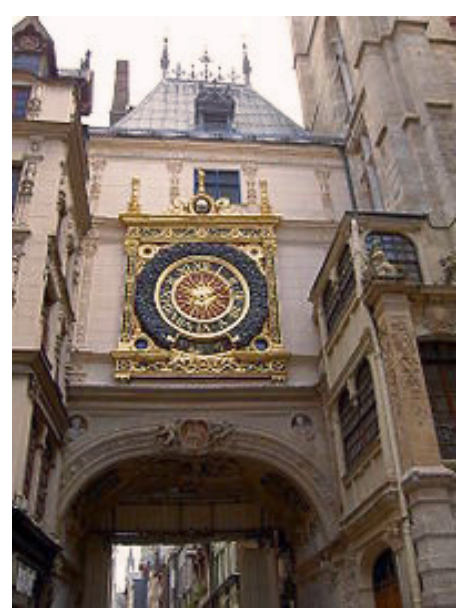

$\vec{u}^{1}$

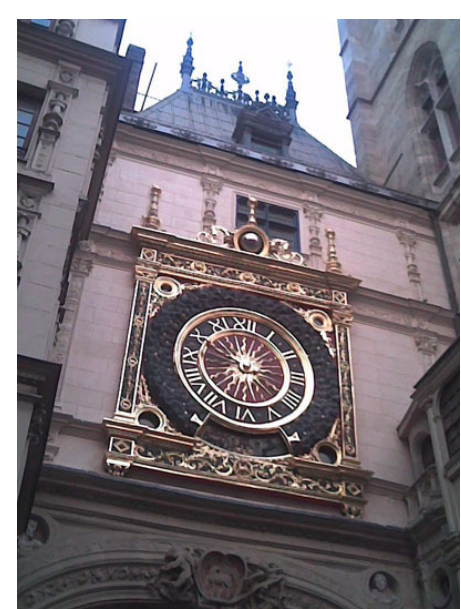

$\vec{U}^{2}$

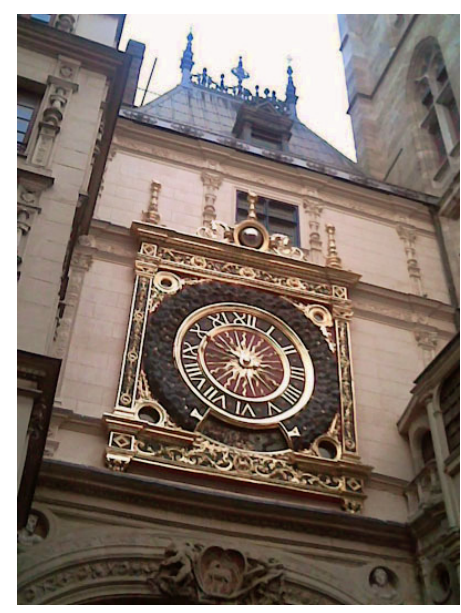

$\vec{u}^{2}$

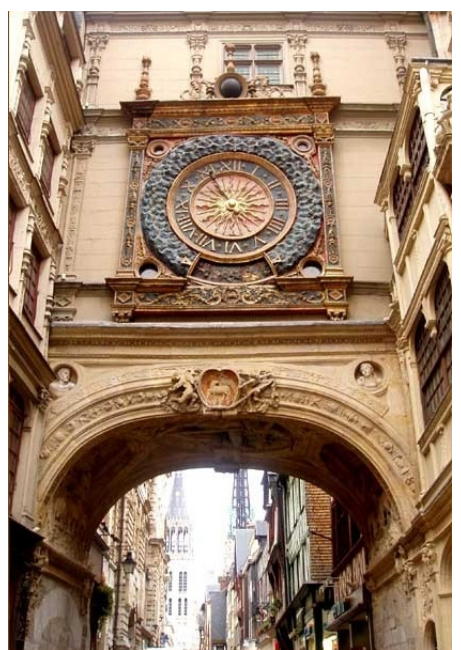

$\vec{U}^{3}$

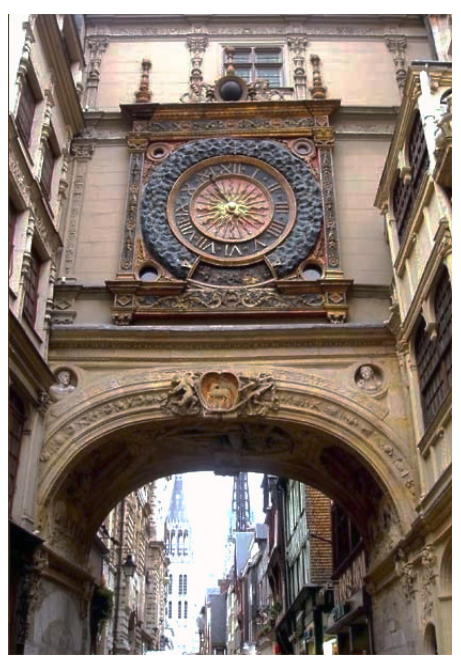

$\vec{u}^{3}$

Fig. 5. Gros Horloge sequence. First line: Original images. Second line: Final images.

\section{ACKNOWLEDGMENTS}

This work was partially funded by Mediapro through the Spanish project CENIT-2007-1012 i3media and by the Centro para el Desarrollo Tecnológico Industrial (CDTI), within the Ingenio 2010 initiative. V. Caselles and E. Provenzi acknowlege partial support by MICINN project, reference MTM2009-08171 and by GRC project, reference 2009 SGR 773, funded by the Generalitat de Catalunya. V. Caselles also acknowledges partial support by IP project "2020 3D Media: Spacial Sound and Vision", Financed by EC, and by "ICREA Acadèmia" prize for excellence in research funded by the Generalitat de Catalunya. E. Provenzi also acknowledges partial support by the Ramón y Cajal fellowship by Ministerio de Ciencia y Tecnología de 
España. N. Papadakis acknowledges support from the Torres Quevedo program of the Ministerio de Educación y Ciencia in Spain.

\section{APPENDIX: THE FIRST VARIATION OF THE HISTOGRAM ENERGY TERMS}

A. The case $N=2$

Let us compute the first variations of the energy (14) with respect to $I_{1}$ and $I_{2}$. We observe that

$$
H_{\vec{I}}(\vec{\lambda})=\frac{1}{|\Omega|} \sum_{x \in \Omega} \mathrm{h}_{\vec{\lambda}}(\vec{I}(x))
$$

where

$$
\mathrm{h}_{\vec{\lambda}}\left(r_{1}, r_{2}\right)=\chi_{\left[0, \lambda_{1}\right]}\left(r_{1}\right) \chi_{\left[0, \lambda_{2}\right]}\left(r_{2}\right) \quad \lambda_{1}, \lambda_{2}, r_{1}, r_{2} \geq 0
$$

Hence, if $\vec{J}=\left(J_{1}, J_{2}\right): \Omega \rightarrow \mathbb{R}^{+} \times \mathbb{R}^{+}$denotes a perturbation, we have

$$
\begin{aligned}
& \left.\frac{d}{d \epsilon}\right|_{\epsilon=0} H_{\vec{I}+\epsilon \vec{J}}(\lambda)=\left.\frac{1}{|\Omega|} \frac{d}{d \epsilon}\right|_{\epsilon=0} \sum_{x \in \Omega} \mathrm{h}_{\vec{\lambda}}(\vec{I}(x)+\epsilon \vec{J}(x))=\left.\frac{1}{|\Omega|} \sum_{x \in \Omega} \frac{d}{d \epsilon}\right|_{\epsilon=0} \mathrm{~h}_{\vec{\lambda}}(\vec{I}(x)+\epsilon \vec{J}(x)) \\
& =-\frac{1}{|\Omega|} \sum_{x \in \Omega}\left[\delta\left(I_{1}(x)-\lambda_{1}\right) \mathrm{h}_{\lambda_{2}}\left(I_{2}(x)\right) J_{1}(x)+\delta\left(I_{2}(x)-\lambda_{2}\right) \mathrm{h}_{\lambda_{1}}\left(I_{1}(x)\right) J_{2}(x)\right] .
\end{aligned}
$$

Then

$$
\begin{aligned}
\left.\frac{d}{d \epsilon}\right|_{\epsilon=0} \mathcal{E}(\vec{I}+\epsilon \vec{J}) \\
=-\frac{1}{|\Omega|} \int_{0}^{1} \int_{0}^{1}\left[H_{\vec{I}}(\vec{\lambda})-H_{\mathrm{ref}}(\vec{\lambda})\right] \sum_{x \in \Omega}\left(\delta\left(I_{1}(x)-\lambda_{1}\right) \mathrm{h}_{\lambda_{2}}\left(I_{2}(x)\right) J_{1}(x)+\delta\left(I_{2}(x)-\lambda_{2}\right) \mathrm{h}_{\lambda_{1}}\left(I_{1}(x)\right) J_{2}(x)\right) d \vec{\lambda} \\
=-\frac{1}{|\Omega|} \sum_{x \in \Omega} \int_{0}^{1} \int_{0}^{1}\left[H_{\vec{I}}(\vec{\lambda})-H_{\mathrm{ref}}(\vec{\lambda})\right]\left(\delta\left(I_{1}(x)-\lambda_{1}\right) \mathrm{h}_{\lambda_{2}}\left(I_{2}(x)\right) J_{1}(x)+\delta\left(I_{2}(x)-\lambda_{2}\right) \mathrm{h}_{\lambda_{1}}\left(I_{1}(x)\right) J_{2}(x)\right) d \vec{\lambda} \\
=-\frac{1}{|\Omega|} \sum_{x \in \Omega}\left(\int_{0}^{1}\left(\int_{0}^{1}\left[H_{\vec{I}}\left(\lambda_{1}, \lambda_{2}\right)-H_{\mathrm{ref}}\left(\lambda_{1}, \lambda_{2}\right)\right] \delta\left(I_{1}(x)-\lambda_{1}\right) d \lambda_{1}\right) \mathrm{h}_{\lambda_{2}}\left(I_{2}(x)\right) d \lambda_{2}\right) J_{1}(x) \\
-\frac{1}{|\Omega|} \sum_{x \in \Omega}\left(\int_{0}^{1}\left(\int_{0}^{1}\left[H_{\vec{I}}\left(\lambda_{1}, \lambda_{2}\right)-H_{\mathrm{ref}}\left(\lambda_{1}, \lambda_{2}\right)\right] \delta\left(I_{2}(x)-\lambda_{2}\right) d \lambda_{2}\right) \mathrm{h}_{\lambda_{1}}\left(I_{1}(x)\right) d \lambda_{1}\right) J_{2}(x) \\
=-\frac{1}{|\Omega|} \sum_{x \in \Omega} \int_{I_{2}(x)}^{1}\left[H_{\vec{I}}\left(I_{1}(x), \lambda_{2}\right)-H_{\mathrm{ref}}\left(I_{1}(x), \lambda_{2}\right)\right] d \lambda_{2} J_{1}(x) \\
-\frac{1}{|\Omega|} \sum_{x \in \Omega} \int_{I_{1}(x)}^{1}\left[H_{\vec{I}}\left(\lambda_{1}, I_{2}(x)\right)-H_{\mathrm{ref}}\left(\lambda_{1}, I_{2}(x)\right)\right] d \lambda_{1} J_{2}(x) .
\end{aligned}
$$


That is,

$$
\begin{aligned}
& \nabla_{I_{1}} \mathcal{E}(\vec{I})=-\frac{1}{|\Omega|} \int_{I_{2}(x)}^{1}\left[H_{\vec{I}}\left(I_{1}(x), \lambda_{2}\right)-H_{\mathrm{ref}}\left(I_{1}(x), \lambda_{2}\right)\right] d \lambda_{2}, \\
& \nabla_{I_{2}} \mathcal{E}(\vec{I})=-\frac{1}{|\Omega|} \int_{I_{1}(x)}^{1}\left[H_{\vec{I}}\left(\lambda_{1}, I_{2}(x)\right)-H_{\mathrm{ref}}\left(\lambda_{1}, I_{2}(x)\right] d \lambda_{1} .\right.
\end{aligned}
$$

Then (16) and (17) follow.

B. The case $N=3$

Let us consider the case $N=3$. Let $\vec{I}=\left(I_{1}, I_{2}, I_{3}\right)$ be a color image defined on the domain $\Omega$ and $H_{\vec{I}}(\vec{\lambda})=\frac{1}{|\Omega|}\left|\left\{x \in \Omega: I_{1}(x) \leq \lambda_{1}, I_{2}(x) \leq \lambda_{3}, I_{3}(x) \leq \lambda_{3}\right\}\right|, \vec{\lambda} \in[0,1]^{3}$. As above, let $H_{\text {ref }}(\vec{\lambda})$ be a specified cumulative histogram. We consider the energy:

$$
\mathcal{E}(\vec{I})=\frac{1}{2} \int_{0}^{1} \int_{0}^{1} \int_{0}^{1}\left(H_{\vec{I}}(\vec{\lambda})-H_{\mathrm{ref}}(\vec{\lambda})\right)^{2} d \vec{\lambda}
$$

Let

$$
\begin{aligned}
\mathcal{H}^{1}\left(i_{1}, i_{2}, i_{3}\right) & \equiv \int_{0}^{i_{2}} \int_{0}^{i_{3}} H\left(i_{1}, \lambda_{2}, \lambda_{3}\right) d \lambda_{2} d \lambda_{3}, \\
\mathcal{H}^{2}\left(i_{1}, i_{2}, i_{3}\right) & \equiv \int_{0}^{i_{1}} \int_{0}^{i_{3}} H\left(\lambda_{1}, i_{2}, \lambda_{3}\right) d \lambda_{1} d \lambda_{3}, \\
\mathcal{H}^{3}\left(i_{1}, i_{2}, i_{3}\right) & \equiv \int_{0}^{i_{1}} \int_{0}^{i_{2}} H\left(\lambda_{1}, \lambda_{2}, i_{3}\right) d \lambda_{1} d \lambda_{2} .
\end{aligned}
$$

With similar computations as in the case $N=2$ we have:

$$
\begin{aligned}
\nabla_{I_{1}} \mathcal{E}(\vec{I})= & -\frac{1}{|\Omega|} \int_{I_{2}(x)}^{1} \int_{I_{3}(x)}^{1}\left[H_{\vec{I}}\left(I_{1}(x), \lambda_{2}, \lambda_{3}\right)-H_{r e f}\left(I_{1}(x), \lambda_{2}, \lambda_{3}\right)\right] d \lambda_{3} d \lambda_{2} \\
= & -\frac{1}{|\Omega|}\left[\mathcal{H}_{\vec{I}}^{1}\left(I_{1}(x), 1,1\right)-\mathcal{H}_{\mathrm{ref}}^{1}\left(I_{1}(x), 1,1\right)\right] \\
& +\frac{1}{|\Omega|}\left[\mathcal{H}_{\vec{I}}^{1}\left(I_{1}(x), I_{2}(x), 1\right)-\mathcal{H}_{\mathrm{ref}}^{1}\left(I_{1}(x), I_{2}(x), 1\right)\right] \\
& +\frac{1}{|\Omega|}\left[\mathcal{H}_{\vec{I}}^{1}\left(I_{1}(x), 1, I_{3}(x)\right)-\mathcal{H}_{\mathrm{ref}}^{1}\left(I_{1}(x), 1, I_{3}(x)\right)\right] \\
& -\frac{1}{|\Omega|}\left[\mathcal{H}_{\vec{I}}^{1}\left(I_{1}(x), I_{2}(x), I_{3}(x)\right)-\mathcal{H}_{\mathrm{ref}}^{1}\left(I_{1}(x), I_{2}(x), I_{3}(x)\right)\right],
\end{aligned}
$$




$$
\begin{aligned}
\nabla_{I_{2}} \mathcal{E}(\vec{I})= & -\frac{1}{|\Omega|} \int_{I_{1}(x)}^{1} \int_{I_{3}(x)}^{1}\left[H_{\vec{I}}\left(\lambda_{1}, I_{2}(x), \lambda_{3}\right)-H_{r e f}\left(\lambda_{1}, I_{2}(x), \lambda_{3}\right)\right] d \lambda_{3} d \lambda_{1} \\
= & -\frac{1}{|\Omega|}\left[\mathcal{H}_{\vec{I}}^{2}\left(1, I_{2}(x), 1\right)-\mathcal{H}_{\mathrm{ref}}^{2}\left(1, I_{2}(x), 1\right)\right] \\
& +\frac{1}{|\Omega|}\left[\mathcal{H}_{\vec{I}}^{2}\left(I_{1}(x), I_{2}(x), 1\right)-\mathcal{H}_{\mathrm{ref}}^{2}\left(I_{1}(x), I_{2}(x), 1\right)\right] \\
& +\frac{1}{|\Omega|}\left[\mathcal{H}_{\vec{I}}^{2}\left(1, I_{2}(x), I_{3}(x)\right)-\mathcal{H}_{\mathrm{ref}}^{2}\left(1, I_{2}(x), I_{3}(x)\right)\right] \\
& -\frac{1}{|\Omega|}\left[\mathcal{H}_{\vec{I}}^{2}\left(I_{1}(x), I_{2}(x), I_{3}(x)\right)-\mathcal{H}_{\mathrm{ref}}^{2}\left(I_{1}(x), I_{2}(x), I_{3}(x)\right)\right], \\
\nabla_{I_{3}} \mathcal{E}(\vec{I})= & -\frac{1}{|\Omega|} \int_{I_{1}(x)}^{1} \int_{I_{2}(x)}^{1}\left[H_{\vec{I}}\left(\lambda_{1}, \lambda_{2}, I_{3}(x)\right)-H_{r e f}\left(\lambda_{1}, \lambda_{2}, I_{3}(x)\right)\right] d \lambda_{2} d \lambda_{1} \\
= & -\frac{1}{|\Omega|}\left[\mathcal{H}_{\vec{I}}^{3}\left(I_{1}(x), 1,1\right)-\mathcal{H}_{\mathrm{ref}}^{3}\left(I_{1}(x), 1,1\right)\right] \\
& +\frac{1}{|\Omega|}\left[\mathcal{H}_{\vec{I}}^{3}\left(1, I_{2}(x), I_{3}(x)\right)-\mathcal{H}_{\mathrm{ref}}^{3}\left(1, I_{2}(x), I_{3}(x)\right)\right] \\
& +\frac{1}{|\Omega|}\left[\mathcal{H}_{\vec{I}}^{3}\left(I_{1}(x), 1, I_{3}(x)\right)-\mathcal{H}_{\mathrm{ref}}^{3}\left(I_{1}(x), 1, I_{3}(x)\right)\right] \\
& -\frac{1}{|\Omega|}\left[\mathcal{H}_{\vec{I}}^{3}\left(I_{1}(x), I_{2}(x), I_{3}(x)\right)-\mathcal{H}_{\mathrm{ref}}^{3}\left(I_{1}(x), I_{2}(x), I_{3}(x)\right)\right] . \\
&
\end{aligned}
$$

\section{REFERENCES}

[1] E. Reinhard, M. Ashikhmin, B. Gooch, and P. Shirley, "Color transfer between images," IEEE Computer Graphics and Applications, vol. 21, pp. 34-41, 2001.

[2] A. Abadpour and S. Kasaei, "A fast and efficient fuzzy color transfer method," in IEEE Symposium on Signal Processing and Information Technology, 2004, pp. 491-494.

[3] J. Morovic and P. Sun, “Accurate 3D image colour histogram transformation," Pattern Recognition Letters, vol. 24, no. 11, pp. 1725-1735, 2003.

[4] L. Neumann and A. Neumann, "Color style transfer techniques using hue, lightness and saturation histogram matching," Computational Aesthetics in Graphics, Visaulization and Imaging 2005, pp. 111-122, 2005.

[5] F. Pitie, A. Kokaram, and R. Dahyot, "N-dimensional probability density function transfer and its application to colour transfer," in International Conference on Computer Vision (ICCV'05), 2005.

[6] F. Pitié, A. Kokaram, and R. Dahyot, "Automated colour grading using colour distribution transfer," Comput. Vis. Image Underst., vol. 107, no. 1-2, pp. 123-137, 2007.

[7] C. Wang and Y. Huang, "A novel color transfer algorithm for image sequences," Journal of Information Science and Engineering, vol. 20, pp. 1039-1056, 2004.

[8] H. Kotera, "A scene-referred color transfer for pleasant imaging on display," in IEEE International Conference on Image Processing (ICIP 2005), vol. 2, 2005, pp. 5-8.

[9] F. Pitié, "Statistical signal processing techniques for visual post-production," Ph.D. Thesis, University of Dublin, Trinity College, 2007.

[10] M. Grundland and N. Dodgson, "Color histogram specification by histogram warping," in Proceedings of SPIE, vol. 5667, 2005, pp. 610-621. 
[11] E. Pichon, M. Niethammer, and G. Sapiro, "Color histogram equalization through mesh deformation," in IEEE International Conference on Image Processing (ICCV' 03), vol. 2, 2003, pp. 117-120.

[12] J. Delon, "Fine comparison of images and other problems," Ph.D. Thesis, École Normale Supérieure de Cachan, 2004.

[13] F. Pitié and A. Kokaram, "The Linear Monge-Kantorovitch Colour Mapping for Example-Based Colour Transfer," in IEEE European Conference on Visual Media Production (CVMP'06), 2007.

[14] C. Villani, Topics in optimal transportation. American Mathematical Society, 2003.

[15] J. Delon, "Midway image equalization," J. Math. Imaging Vis., vol. 21, no. 2, pp. 119-134, 2004.

[16] I. Cox, S. Roy, and S. Hingorani, "Dynamic histogram warping of image pairs for constant image brightness," in IEEE International Conference on Image Processing (ICIP'95), vol. 2, 1995, pp. 366-369.

[17] C. Ballester, V. Caselles, L. Igual, J. Verdera, and B. Rougé, “A variational model for p+ xs image fusion,” International Journal of Computer Vision, vol. 69, no. 1, pp. 43-58, 2006. 